\title{
ON THE CRITICAL BEHAVIOUR AND PHASE DIAGRAM OF ISING-LIKE METAMAGNET IN A TRANSVERSE FIELD
}

\author{
K. LUKIERSKA-WALASEK \\ Institute of Physics, Pedagogical University of Zielona Góra \\ P1. Słowiański 6, 65-065 Zielona Góra, Poland
}

The quantum Ising-like metamagnet in a field $\Gamma$ perpendicular to the easy direction in the mean field approximation is investigated. The conditions for the existence of critical and tricritical points have been analyzed. The result is illustrated by an appropriate plot in three-dimensional space of the following parameters: the temperature, $\Gamma$, and field parallel to the easy direction.

PACS numbers: $75.30 . \mathrm{Kz}, 81.30 . \mathrm{Dz}, 05.70 . \mathrm{Fh}$

A behaviour of an Ising system in a transverse magnetic field $\Gamma$ (applied to the easy direction) has been the subject of several theoretical [1-4] and experimental studies $[5,6]$. An important feature of such system is a crossover near the zero-temperature from the quantum to the classical behaviour. It has been studied theoretically [2-4] and observed in a simple two-sublattice Ising-type antiferromagnet $\mathrm{MnCl}_{2} \cdot 4 \mathrm{H}_{2} \mathrm{O}\left(\mathrm{MnCl}_{2} \cdot 4 \mathrm{D}_{2} \mathrm{O}\right)$ at temperatures in a range $100<T<350 \mathrm{mK}$ $[5,6]$.

It is interesting, however, to consider the Ising-type two-sublattice system, when except of the transverse field $\Gamma$ a field $H$ parallel to the easy direction is applied. In such a case we have metamagnet, in which the phase transition can be driven by three parameters, namely, $\Gamma, H$, and $T$. This problem has been recently reported in Ref. [7], where the system in the quantum regime has been investigated.

The aim of the present paper is to show that in the three-dimensional space of parameters $\Gamma, H$, and $T$ not only critical lines, in $\Gamma, H$ [7] and $T, H$ [8]-planes but also a surface of critical points exist, which is bounded by the line of tricritical points.

Consider an Ising-like metamagnet described by the following Hamiltonian [7] (cf. also Ref. [8]): 


$$
\begin{aligned}
\mathcal{H}= & -\frac{1}{2} \sum_{a, a^{\prime}} J_{a a^{\prime}} S_{a}^{z} S_{a^{\prime}}^{z}-\frac{1}{2} \sum_{b, b^{\prime}} J_{b b^{\prime}} S_{b}^{z} S_{b^{\prime}}^{z}+\frac{1}{2} \sum_{a, b} K_{a, b} S_{a}^{z} S_{b}^{z} \\
& -H\left(\sum_{a} S_{a}^{z}+\sum_{b} S_{b}^{z}\right)-\Gamma\left(\sum_{a} S_{a}^{x}+\sum_{b} S_{b}^{x}\right),
\end{aligned}
$$

where $S_{i}^{\mu}$ with $\mu=x, z$ denotes the $\mu$-component of the spin operator referred to the site $i=a, b$. The spins occupy the sites of two identical sublattices $A$ and $B$, labelled by $a, a^{\prime} \in A$ and $b, b^{\prime} \in B$. The exchange interactions $J_{i j}$ within each sublattice are ferromagnetic, while the inter-sublattice interactions $K_{i j}$ are antiferromagnetic. The spin $S$ is assumed to be arbitrary. In Eq. (1) the term with the staggered field $H^{\dagger}[7,8]$ has been omitted because one assumes that $H^{\dagger}=0$.

The partition function $Z=\operatorname{Tr} \exp (-\beta \mathcal{H})$ can be represented as the following functional integral [7]:

$$
Z=\int \mathrm{d}(\varphi) \exp (-S[\varphi])
$$

where $S[\varphi]$ is the functional Hamiltonian [7] and $\varphi_{i}(\tau)$ denotes the order parameter field proportional to the local staggered magnetization dependent on the Matsubara parameter $\tau(0<\tau<\beta)$. The presence of the $\tau$-parameter is a consequence of noncommutativity of the spin operators entering Hamiltonian $(1)[3,7]$.

The part of $S[\varphi]$ relevant for the description of the critical behaviour of the system reads [7]:

$$
\begin{aligned}
S_{\text {eff }} & =\frac{1}{2} \int \mathrm{d}^{d} r \int_{0}^{\beta} \mathrm{d} \tau\left[(\nabla \varphi)^{2}+c\left(\frac{\partial \varphi}{\partial \tau}\right)^{2}+r_{0} \varphi^{2}\right] \\
& +\frac{u_{0}}{4 !} \int \mathrm{d}^{d} r \int_{0}^{\beta} \mathrm{d} \tau \varphi^{4}-\frac{v_{0}}{2} \int \mathrm{d}^{d} r\left(\int_{0}^{\beta} \mathrm{d} \tau \varphi\right)^{2},
\end{aligned}
$$

where $\varphi \equiv \varphi(r, \tau)$ is the appropriately rescaled (cf. Ref. [3]) field $\varphi_{i}(\tau)$ in the continuum limit with $r$ denoting $d$-dimensional spatial vector. Because of the limited space available for the present work we will not define here the parameters $c, r_{0}$, $u_{0}$, and $v_{0}$ referring to the paper [7].

In the mean-field approximation we evaluate $Z(2)$ by the saddle point method. This leads to the following Landau expansion of the free energy:

$$
\frac{F}{V}=-\frac{k_{\mathrm{B}} T}{V} \ln Z=\frac{\tilde{r}_{0}}{2} M^{2}+\frac{u_{0}}{4 !} M^{4}+\mathrm{O}\left(M^{6}\right),
$$

where $M=\langle\varphi(r, \tau)\rangle_{S}$ with $\langle\ldots\rangle_{S}=\int \mathrm{d}(\varphi) \exp (-S[\varphi]) \ldots / \int \mathrm{d}(\varphi) \exp (-S[\varphi])$ is the order parameter, $V$ denotes the volume of the system, and

$$
\tilde{r}_{0}=r_{0}-\beta v_{0} \text {. }
$$

As usual, at the critical point $\partial^{2} F /\left.\partial M^{2}\right|_{M=0}=0$ and $\partial^{4} /\left.\partial M^{4}\right|_{M=0}>0$. Therefore, a condition for a critical point reads

$$
\begin{aligned}
& \tilde{r}_{0}(T, \Gamma, H)=0, \\
& u_{0}(T, \Gamma, H)>0,
\end{aligned}
$$


whereas for a tricritical point we have

$$
\begin{aligned}
& \tilde{r}_{0}(T, \Gamma, H)=0, \\
& u_{0}(T, \Gamma, H)=0 .
\end{aligned}
$$

Equations (5) and (6) were analyzed for $S=1 / 2$. It is rather easy to find the solutions of (5) and (6) in an explicit form in two limiting cases: $\Gamma=0$ and $T=0$. For $\Gamma=0$ one obtains in the $(T, H)$-plane a line of critical points, which satisfies the equation (cf. Ref. [8]):

$$
\bar{H}=2 \bar{T} \tanh ^{-1}\left[(1-2 \bar{T})^{1 / 2}\right],
$$

which terminates in the tricritical point $\bar{T}=0.3333$ and $\bar{H}=0.4390$, where $\bar{T}$ and $\bar{H}$ denote the temperature and longitudinal field scaled by the Néel temperature $T_{0}=I_{0} / 2$. In the quantum regime for $T=0$ the equation of the critical line in $(\Gamma, H)$-plane is following [7]:

$$
\bar{H}=\bar{\Gamma}^{2 / 3}\left(1-\bar{\Gamma}^{2 / 3}\right)^{1 / 2}
$$

and the tricritical point is located at $\bar{\Gamma}=0.7155$ and $\bar{H}=0.3578$, where $\bar{\Gamma}=\Gamma / T_{0}$.

For arbitrary parameters $T, \Gamma, H$ Eqs. (5) and (6) can be solved only numerically. Our calculations give that the surface of critical points, bounded by the line of the tricritical points exists. In addition, there is the isolated critical line at $T=0$ terminating in the tricritical point (cf. [7]).

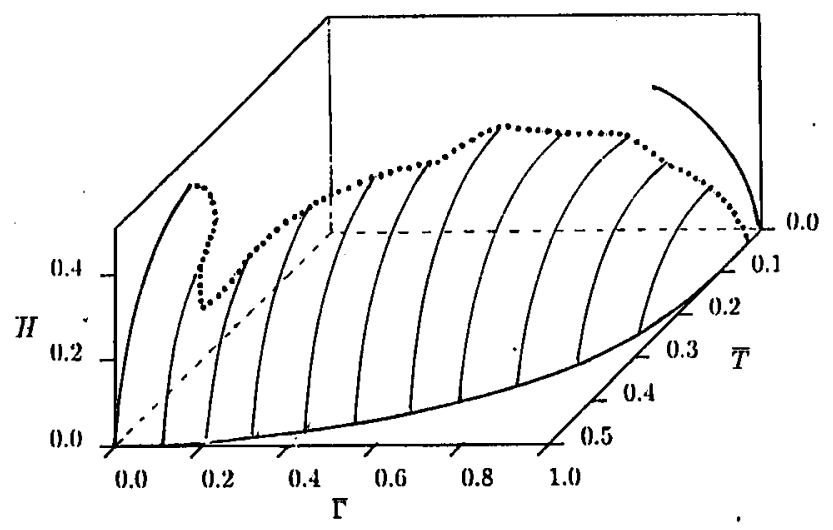

Fig. 1. Critical surface in the space of parameters $\bar{H}, \bar{\Gamma}$ and $\bar{T}$, which are, respectively, the temperature $k_{\mathrm{B}} T$, parallel $(H)$ and transverse $(\Gamma)$ fields scaled by the Néel temperature $T_{0}$. The loci of tricritical points are indicated by the dotted line. In the $\bar{\Gamma}, \bar{H}$-plane at $\bar{T}=0$ the isolated critical line exists.

The results are presented in Fig. 1. For the points $(\bar{H}, \bar{\Gamma}, \bar{T})$ lying under the critical surface the ordered phase exists with $M \neq 0$, while above we have the phase with $M=0$. Lines and surfaces of coexistence of the phases are not placed, because we are interested only in a continuous transition. 


\section{References}

[1] M. Suzuki, Prog. Theor. Phys. 56, 1454 (1976) and references cited therein.

[2] I.D. Lawrie, J. Phys. C 11, 1123 (1978); ibid. 11, 3858 (1978).

[3] K. Lukierska-Walasek, K. Walasek, J. Phys. C 16, 3149 (1983).

[4] K. Lukierska-Walasek, W. Salejda, Phys. Lett. A 111, 415 (1985).

[5] J.J. M. Steijger, E. Frikkee, L.J. de Jongh, W.J. Huiskamp, J. Magn. Magn. Mater. 31-34, 1091 (1983).

[6] W.A.C. Erkelens, L.P. Regnault, J. Rossat-Mignod, J.E. Moore, R.A. Butera, Europhys. Lett. 1, 37 (1986).

[7] K. Lukierska-Walasek, Phys. Lett. A 171, 423 (1992).

[8] I.D. Lawrie, D. Sarbach, in: Phase Transitions and Critical Phenomena, Vol. 9, Eds. C. Domb, J. Lebowitz, Academic Press, New York 1984, p. 115. 